ज्ञ FRANÇAISE

$\supset \mathrm{DE}$

때 PÉDAGOGIE

\section{Revue française de pédagogie}

Recherches en éducation

164 | juillet-septembre 2008

Évaluation des politiques éducatives et comparaisons internationales

\title{
Composition académique et sociale des établissements, efficacité et inégalités scolaires : une comparaison internationale
}

Academic and social elements on the school efficiency and school inequality: an international comparison

Composición académica y social de los establecimientos, eficacia y desigualdades escolares: una comparación internacional

Akademische und soziale Zusammensetzung der Schulanstalten, Effizienz und Ungleichheiten in der Schule: ein internationaler Vergleich

\section{Christian Monseur et Marcel Crahay}

\section{OpenEdition}

Journals

\section{Édition électronique}

URL : http://journals.openedition.org/rfp/2128

DOI : $10.4000 /$ rfp. 2128

ISSN : 2105-2913

\section{Éditeur}

ENS Éditions

Édition imprimée

Date de publication : 1 juillet 2008

Pagination : 55-66

ISBN : 978-2-7342-1136-5

ISSN : 0556-7807

\section{Référence électronique}

Christian Monseur et Marcel Crahay, «Composition académique et sociale des établissements, efficacité et inégalités scolaires : une comparaison internationale ", Revue française de pédagogie [En ligne], 164 | juillet-septembre 2008, mis en ligne le 01 juillet 2012, consulté le 30 avril 2019. URL: http://journals.openedition.org/rfp/2128; DOI : 10.4000/rfp.2128 


\section{Composition académique et sociale des établissements, efficacité et inégalités scolaires: une comparaison internationale}

Christian Monseur et Marcel Crahay

L'article aborde les effets de l'agrégation sociale et de l'agrégation académique des élèves sur l'efficacité et l'équité des systèmes d'enseignement. Diverses analyses secondaires des bases de données PISA 2000, 2003 et 2006 permettent d'aboutir à plusieurs constats qui devraient interpeller certains responsables des politiques éducatives. Précisément, l'article montre que plus un pays recourt au regroupement homogène des élèves selon des critères sociaux et/ou académiques:

- plus le bénéfice d'un élève défavorisé de fréquenter une école favorisée sera important;

- plus le déficit d'un élève défavorisé de fréquenter une école défavorisée sera important;

- plus l'écart de performance qui sépare les élèves faibles et les élèves forts sera élevé ;

- plus l'écart de performance qui sépare les élèves défavorisés et les élèves favorisés sera élevé ;

- plus la corrélation entre origine sociale et performance sera élevée ;

- plus la performance moyenne en lecture sera faible et dans une moindre mesure.

Descripteurs (TEE) : hétérogénéité des élèves, équité et efficacité des systèmes d'enseignement, effet d'agrégation, politiques éducatives.

\section{ÉDUCATION COMPARÉE ET INÉGALITÉS SOCIALES DE PERFORMANCES SCOLAIRES}

Les inégalités sociales de réussite à l'école sont connues. Depuis le début des années cinquante, les enquêtes sociologiques n'ont cessé de montrer que les enfants dont les parents ont un statut socioéconomique (SSE (1)) inférieur accèdent moins souvent aux niveaux les plus élevés du cursus scolaire que ceux dont les parents ont un SSE supérieur. À cet égard, le rapport Coleman aux États-Unis (Coleman et al., 1966) a joué un rôle étendard, même si on dispose, pour plusieurs autres pays, d'études équivalentes datant de la même période. Depuis cette époque, les mêmes constats se répètent: d'importantes inégalités de réussite scolaire sont liées à l'origine sociale des élèves. De surcroît, ce phénomène concerne de nombreux pays, si l'on en croit les résultats des études internationales. Ainsi, la corrélation entre SSE et performance est particulièrement élevée 
dans les pays dits développés et s'est amplifiée entre 1970-1971 et les derniers surveys (Keeves, 1992). Cependant, si le phénomène est transnational, son amplitude varie considérablement d'un pays à l'autre (OCDE, 2001, 2004 \& 2007).

Cette observation laisse supposer que l'ampleur du phénomène est un facteur modifiable. Dans cet article, on fera l'hypothèse générale que certaines variables scolaires et contextuelles font partie des éléments qui affectent l'importance des inégalités sociales de résultats. Plus précisément, l'objectif des analyses secondaires des données PISA présentées ici est d'illustrer comment l'éducation comparée peut contribuer à l'identification des mécanismes structurels par lesquels l'école participe à la production d'inégalités sociales de réussite scolaire. Le premier axe du présent article consiste à examiner dans quelle mesure certains de ces mécanismes, tels que la tendance au regroupement académique ou social, sont communs à tous les systèmes éducatifs ou, au contraire, s'ils sont propres à un (ou des) sousensemble(s). Le second axe d'analyse sera d'examiner les conséquences de ces mécanismes structurels de production des inégalités en termes d'équité et d'efficacité des systèmes éducatifs. En effet, l'adoption de stratégies visant à réduire l'impact de l'origine sociale sur les acquis scolaires peut être freinée par la crainte de conséquences négatives en termes de rendement. II est donc légitime de poser la question du bénéfice général sur le rendement scolaire de choix structurels, fussent-ils reconnus comme inéquitables, afin d'éclairer au mieux le débat sur les politiques d'éducation.

\section{Agrégation sociale et agrégation académique}

Les sources d'inégalités peuvent être multiples. Trois sont régulièrement mises en évidence par les études à grande échelle menées par I'IEA (Association for the Evaluation of Educational Achievement) et par l'OCDE :

1) Le SSE de la famille des élèves ;

2) La langue parlée à la maison, qui peut ou non correspondre à celle utilisée pour l'enseignement ;

3) Le fait d'être ou non natif du pays dans lequel l'enseignement est reçu.

Ces trois paramètres ne sont pas indépendants. En effet, en raison de la nature des phénomènes migratoires, bon nombre d'immigrés sont de SSE modeste. Ainsi, que ce soit au départ des données de PISA 2000 (Crahay \& Monseur, 2006) ou des données de PISA 2006 (2), on note que dans tous les pays de l'OCDE, il y a un effet d'interaction entre les variables SSE, d'une part, et les variables « langue » et « natif ». Dès lors que le SSE est tenu sous contrôle, le poids des deux autres variables est négligeable.

En termes de politique éducative, l'intérêt stratégique de ces constats va de soi. Dans la mesure où le SSE apparaît comme la variable la plus déterminante, il est rationnel de se soucier principalement du " contrôle " des effets de cette variable. Dans le cas contraire, au cas où ces hypothèses auraient été réfutées ou seulement confirmées dans certains pays, la réflexion sur les stratégies de lutte contre les inégalités en aurait été rendue plus complexe encore et, surtout, dépendante des contextes nationaux. Par ailleurs, il est intéressant de souligner que la prépondérance du SSE se confirme, que l'on prenne pour critère la compréhension en lecture (PISA 2000) ou les connaissances scientifiques (PISA 2006). Cette confirmation est cruciale à nos yeux : l'importance du SSE étant indépendante de la discipline concernée, il devient légitime d'espérer un bénéfice général sur le rendement scolaire des stratégies visant à réduire les effets délétères de l'origine sociale des élèves sur la réussite scolaire.

Parmi les facteurs d'inégalité liés à l'organisation des écoles, on peut pointer les caractéristiques de la population fréquentant l'établissement. Déjà en 1966, Coleman et al. (1966) avaient mis en évidence que « les caractéristiques des autres élèves rendent compte de plus de variations dans la réussite des élèves issus des minorités que n'importe quelle caractéristique des équipements scolaires et un peu plus que les attitudes du corps enseignant » (1966, p. 303 cité par Cherkaoui, 1979, p. 60).

Le phénomène observé par Coleman et al. (1966) semble avoir une portée plus générale. Réanalysant avec des techniques statistiques plus sophistiquées les données de Coleman et al. (1966), Mayeske et al. (1972) concluent en soulignant la probabilité et l'importance de ce qu'on appelle désormais les effets d'agrégation, c'est-à-dire des effets spécifiques produits sur les performances scolaires par le regroupement d'élèves d'une même catégorie sociale ou ethnique au sein d'un même établissement. Pour ces auteurs, l'effet de l'école ne se distingue pas de l'effet du milieu familial, du simple fait que l'affectation des élèves dans les écoles n'a rien d'aléatoire: les enfants des familles de statut socio-économique supérieur fréquentent les meilleures écoles et cellesci sont meilleures parce qu'elles sont fréquentées par des élèves issus de milieu aisé. 
Des effets d'agrégation ont également été mis en évidence dans plusieurs des premières études de I'IAE (Association for the Evaluation of Education Achievement) (Husén, 1975 ; Keeves, 1992). Plus récemment, Vandenberghe (2001) a montré que la performance des élèves en sciences et en mathématiques est d'autant plus élevée, toutes choses égales par ailleurs, que les compagnons de classe sont de milieu social ou de niveau scolaire élevé. Cet effet lié à la composition sociale ou académique des établissements est parfois aussi fort que l'effet propre de l'origine sociale des élèves (Caldas \& Bankson, 1997, cité par Duru-Bellat, 2003).

Crahay \& Monseur (2006) ont également observé, par l'intermédiaire d'une analyse de la valeur ajoutée, des effets d'agrégation sociale à travers une large diversité de systèmes éducatifs. Leur analyse consiste à analyser les résidus de la prédiction du SSE de l'élève sur sa performance scolaire, en fonction de la composition sociale de l'école. Les résultats attestent que, dans tous les pays, des effets d'agrégation sont observables. Autrement dit, il semble bien que, partout au monde, on constate un effet de la composition socioéconomique des écoles sur les performances en lecture des élèves. Cependant, l'importance de celuici varie selon les pays: ils sont minimes dans des pays comme le Danemark, la Finlande, I'Islande, la Norvège, la Suède, alors qu'ils sont importants dans la plupart des autres pays (Crahay \& Monseur, 2006).

Dans le présent article, nous chercherons à savoir si l'existence d'un effet école, lié au regroupement social des élèves, est également attesté lorsqu'on applique les mêmes analyses aux données PISA 2006 qui, elles, portent sur les compétences scientifiques. II s'agira donc de tester la généralité d'un constat préalablement établi au niveau international pour la compréhension en lecture et de vérifier si, en sciences comme en lecture, la magnitude des phénomènes varie fortement entre pays (hypothèse 1). En effet, ce n'est plus tant l'existence de phénomènes d'agrégation qui fait question désormais, mais bien leur degré de généralité ou d'universalité ainsi que leur variabilité en fonction des systèmes éducatifs. Nous supposons, en effet, que ces phénomènes transcendent les disciplines, mais que leur ampleur variable est due à des pratiques pédagogiques, des caractéristiques d'écoles et des politiques éducatives différentes.

Nos analyses sur l'impact des effets d'agrégation s'inscrivent dans un courant de recherche qu'il est convenu d'appeler le school mix. D'après DuruBellat (2003), ce courant émerge à partir des années quatre-vingt, dans la foulée des travaux sur le school effectiveness qui s'attache à explorer l'effet des caractéristiques propres aux écoles, leur mode de fonctionnement, leurs ressources matérielles, leur climat, leur ethos, la capacité des enseignants à travailler en équipe, le dynamisme du chef d'établissement, etc. II s'agit en quelque sorte de prendre en considération le fait que les classes sont nichées dans des structures plus larges - l'école - et, dans la foulée, de contextualiser l'effet des pratiques d'enseignement, en s'efforçant d'appréhender ce qu'est la part propre à l'environnement matériel et psychosocial de l'établissement. De l'intérieur du courant (cf. notamment Teddle et al., cité par Duru-Bellat, 2003), mais aussi de l'extérieur (d'auteurs critiques tels que Thrupp, 1995, cité par Duru-Bellat, 2003), s'est imposée l'idée qu'il convenait de "contextualiser les facteurs de contexte ", c'est-à-dire de reconnaître que l'établissement est lui-même niché dans un environnement social plus large (la communauté, le quartier, la ville ou le village, etc.). Parmi les paramètres du contexte scolaire, les caractéristiques du public fréquentant l'école sont essentielles. Celles-ci, désignées par les chercheurs anglo-saxons par l'expression school mix, se composent principalement du niveau scolaire moyen des élèves fréquentant l'école ainsi que de la disparité de leurs compétences - ce qu'on nomme l'academic mix - et de leur profil sociologique (moyenne et diversité) - ce qu'on nomme le social mix.

Dans nos analyses, nous distinguerons systématiquement l'academic mix du social mix. Selon des auteurs comme Duru-Bellat (2003), il importe en effet de préciser la notion de school mix et, notamment, de mieux cerner comment l'academic mix et le social mix articulent leurs effets spécifiques. La distinction que nous avons eu le souci d'opérer entre le social mix et l'academic mix ne permet pas véritablement d'analyser l'articulation des deux phénomènes, mais permet néanmoins d'estimer leur prévalence respective sur les aspects de l'équité et de l'efficacité des systèmes d'enseignement que nous analysons.

\section{Impact des agrégations sociale et académique sur l'équité et l'efficacité des systèmes d'enseignement}

Selon la logique de notre raisonnement, les phénomènes d'agrégation des élèves sont préjudiciables aux élèves en difficulté ; ceci devrait se marquer au niveau du rendement général des systèmes d'enseignement et de leur équité. Plus précisément, on peut supposer que, plus le fonctionnement d'un système d'enseignement engendre des phénomènes d'agrégation sociale ou académique, plus il amplifie les différences entre élèves faibles et élèves forts et plus 
il amplifie ainsi les inégalités sociales devant l'école. Ceci devrait rejaillir sur l'efficacité générale des systèmes. En effet, sur la base d'une revue circonstanciée des études internationales (IEA et PISA), Crahay (2000 et 2007) on a pu constater, qu'en règle générale, les systèmes d'enseignement les plus égalitaires sont également les plus efficaces.

Dans l'étude longitudinale qu'elle a consacrée au fonctionnement des collèges français et à leurs effets sur les élèves de sixième, Grisay (1993) fait un constat semblable au niveau d'un système éducatif. Les résultats confortent par ailleurs ceux de Coleman et de I'IEA (Keeves, 1992) : I'hétérogénéité de la population recrutée par le collège s'avère être un facteur positif. Ceci est vrai en ce qui concerne les performances en français et en mathématiques, mais est également vrai en ce qui concerne la réduction des écarts entre élèves faibles et forts. Les élèves des collèges à population hétérogène tendent, après deux ans, à se ressembler davantage ; à l'inverse, les différences de performances s'accentuent dans les collèges dont la population était, au départ, la plus homogène.

Nous pensons que les observations réalisées par Grisay au niveau des collèges français relèvent de phénomènes plus généraux et sont, dès lors, susceptibles d'être observés au niveau d'autres systèmes éducatifs. En d'autres termes, le regroupement homogène des élèves accentue la diversité des performances, que celles-ci soient appréhendées au niveau établissement ou au niveau du pays. Ainsi, l'écart qui sépare les élèves de SSE inférieur et supérieur, socialement, devrait être d'autant plus élevé que les systèmes éducatifs se caractérisent par d'importants phénomènes d'agrégation.

Précisément, nous suspectons que, plus un système éducatif recourt à l'agrégation sociale et/ou académique :

- plus la dispersion des performances scolaires sera élevée ou, dit autrement, plus l'écart qui sépare les élèves faibles des élèves forts sera important (hypothèse 2a) ;

- plus l'écart de performance entre élèves de SSE inférieur et supérieur sera élevé (hypothèse $2 b$ ).

Enfin, considérant que (1) les pays caractérisés par d'importants phénomènes d'agrégation sociale et/ou académique utilisent différentes pratiques pédagogiques ségrégatives telles que le redoublement, la différentiation précoce de l'enseignement, les classes de niveaux, etc., et que (2) ces différentes pratiques pédagogiques corrèlent avec le SSE des élèves, nous formulons une dernière hypothèse : dans les systè- mes éducatifs, le déterminisme social (exprimé par la corrélation entre le SSE de l'élève et sa performance scolaire) sera d'autant plus élevé qu'est importante l'agrégation sociale et académique (hypothèse $2 c$ ).

Enfin, nous reprendrons à notre compte l'hypothèse générale selon laquelle les systèmes les plus égalitaires sont aussi les plus efficaces. On complétera donc les hypothèses précédentes en supposant l'existence de liens et, plus précisément, de corrélations négatives entre les taux d'agrégation sociale et académique des systèmes d'enseignement et leur rendement (hypothèse 3 ).

\section{Effets de la composition sociale des écoles sur la performance scolaire des élèves}

L'hypothèse 1 présuppose l'existence, dans tous les pays ayant participé à PISA 2006, d'écoles socialement différenciées en fonction de la population d'élèves qui les fréquentent. Ceci n'est pas difficile à établir. Pour ce faire, nous avons, dans un premier temps, réparti les écoles en trois catégories, dénommées "écoles défavorisées ", "écoles mixtes " et "écoles favorisées ", selon que le SSE moyen des élèves qui fréquentent l'établissement diffère ou non statistiquement de la moyenne nationale (3). La figure 1 présente le pourcentage d'écoles défavorisées, mixtes et favorisées dans les différents systèmes éducatifs. Les pays y sont classés par ordre décroissant d'écoles mixtes.

À une extrémité, on trouve principalement les pays de l'Europe du Nord et, dans une moindre mesure, les pays anglo-saxons, à l'exception de l'Australie. Dans ces pays, les écoles mixtes constituent la norme. À l'opposé, on trouve des pays comme la France, la Pologne, la Corée, la Belgique, l'Australie, le Japon, la Hongrie et le Luxembourg où les écoles mixtes correspondent à moins de $40 \%$.

Pour procéder à la mise sous épreuve de l'hypothèse 1 , nous avons, d'une part, tenu compte de la répartition des écoles en trois catégories (écoles défavorisées, mixtes et favorisées) et, d'autre part, nous avons réparti les élèves en trois catégories: les $25 \%$ d'élèves les plus défavorisés, les $50 \%$ intermédiaires et les $25 \%$ d'élèves les plus favorisés.

Par pays, il a été procédé à une analyse de régression, tous élèves confondus et toutes écoles confondues, de sorte à prédire les scores au test de science à partir du SSE. Ce qui nous intéressera ici, ce sont les résidus et, plus particulièrement, leur analyse en fonction du type d'élèves et en fonction du type d'écoles. Le tableau 1 est à interpréter en tenant compte de 
Figure 1. - Pourcentage d'écoles défavorisées, mixtes et favorisées (OCDE, PISA 2006)

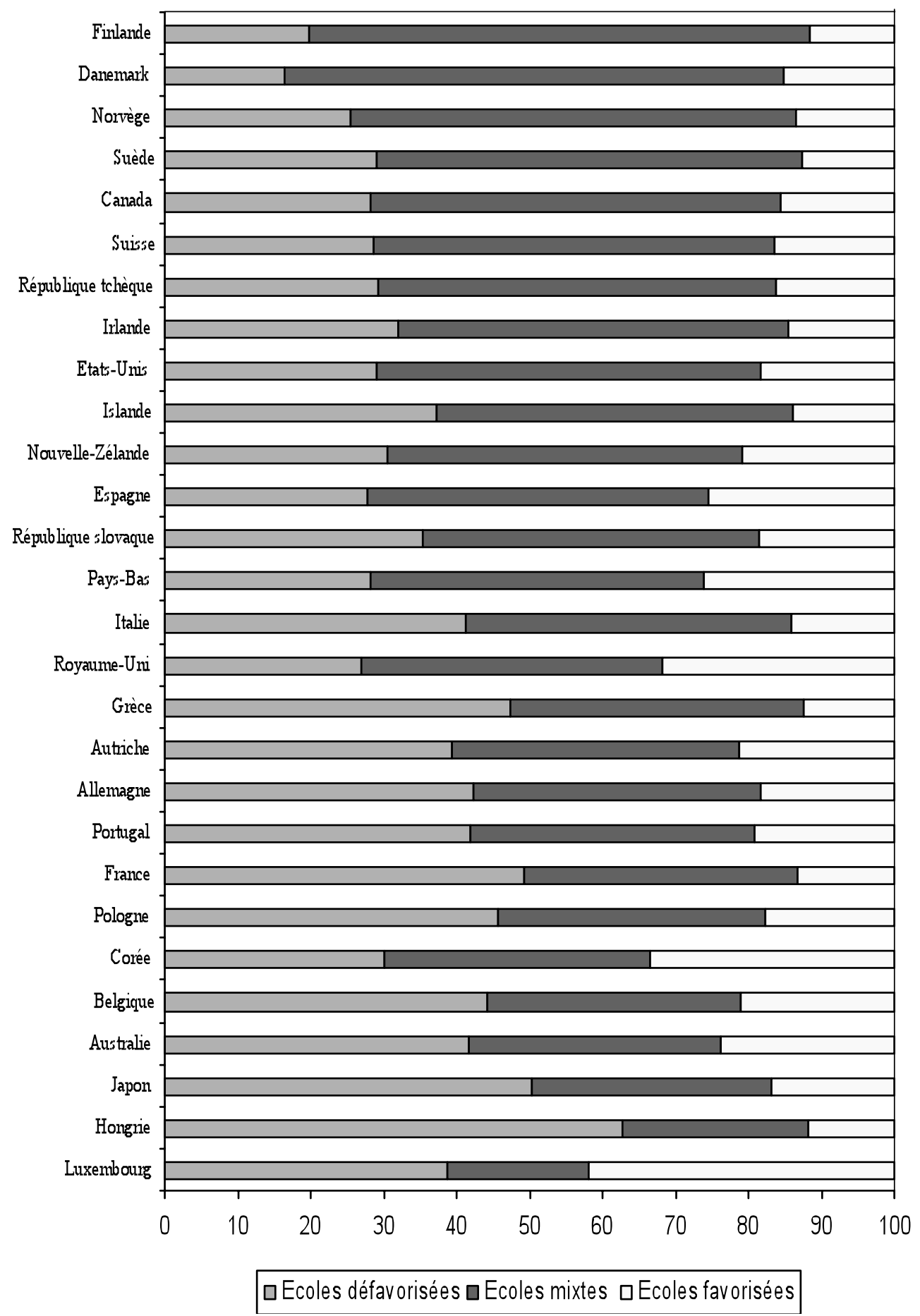


Tableau 1. - Scores résiduels moyens des élèves de SSE inférieur et supérieur en fonction du type d'école fréquentée

\begin{tabular}{|c|c|c|c|c|c|c|}
\hline & \multicolumn{2}{|c|}{ Écoles défavorisées } & \multicolumn{2}{|c|}{ Écoles mixtes } & \multicolumn{2}{|c|}{ Écoles favorisées } \\
\hline & $\begin{array}{l}\text { Élèves } \\
\text { défavorisés }\end{array}$ & $\begin{array}{l}\text { Élèves } \\
\text { favorisés }\end{array}$ & $\begin{array}{c}\text { Élèves } \\
\text { défavorisés }\end{array}$ & $\begin{array}{c}\text { Élèves } \\
\text { favorisés }\end{array}$ & $\begin{array}{c}\text { Élèves } \\
\text { défavorisés }\end{array}$ & $\begin{array}{c}\text { Élèves } \\
\text { favorisés }\end{array}$ \\
\hline Finlande & & & & & & 16,4 \\
\hline Norvège & & & & & & 13,2 \\
\hline Suède & & & & & & 20,6 \\
\hline Danemark & $-15,7$ & $-20,1$ & & & 20,9 & 16,3 \\
\hline \multicolumn{7}{|l|}{ Islande } \\
\hline Royaume-Uni & & $-50,1$ & & $-14,8$ & 35,3 & 27,4 \\
\hline Suisse & $-15,6$ & $-33,8$ & & $-27,7$ & 104,3 & 32,2 \\
\hline Canada & $-15,0$ & $-32,6$ & 7,6 & & 24,5 & 9,6 \\
\hline $\begin{array}{l}\text { Nouvelle- } \\
\text { Zélande }\end{array}$ & & & & & 46,2 & 16,5 \\
\hline Irlande & $-21,8$ & & 13,8 & & & 9,2 \\
\hline Luxembourg & $-17,5$ & $-59,2$ & 20,6 & & 57,5 & 17,4 \\
\hline Pays-Bas & $-40,3$ & $-98,8$ & 28,7 & $-15,0$ & 97,1 & 26,7 \\
\hline Australie & $-6,3$ & $-32,8$ & & $-13,1$ & 35,0 & 17,7 \\
\hline Espagne & & $-22,6$ & & $-11,7$ & 24,6 & $-8,8$ \\
\hline Italie & $-21,7$ & $-70,5$ & & $-35,9$ & 76,1 & 28,5 \\
\hline Japon & $-36,4$ & $-93,1$ & 27,4 & $-18,6$ & 72,7 & 20,4 \\
\hline \multicolumn{7}{|l|}{ Pologne } \\
\hline Corée & $-19,5$ & $-63,4$ & 30,6 & & 46,0 & 19,2 \\
\hline Allemagne & $-39,6$ & $-113,0$ & 25,2 & $-27,8$ & 99,1 & 29,2 \\
\hline Belgique & $-32,3$ & - 77,7 & 30,8 & $-25,0$ & 81,3 & 24,2 \\
\hline États-Unis & & $-41,7$ & 15,6 & & 24,5 & 22,6 \\
\hline $\begin{array}{l}\text { République } \\
\text { tchèque }\end{array}$ & $-21,6$ & $-89,9$ & 12,3 & $-30,3$ & 67,2 & 41,5 \\
\hline Autriche & $-33,5$ & $-80,8$ & 24,1 & $-13,3$ & 82,5 & 12,8 \\
\hline France & $-34,0$ & $-118,9$ & 41,8 & & 97,0 & 24,1 \\
\hline Portugal & & $-26,4$ & & $-11,8$ & 48,9 & 14,4 \\
\hline Grèce & $-21,5$ & $-47,0$ & 21,0 & $-12,6$ & 28,8 & 13,0 \\
\hline $\begin{array}{l}\text { République } \\
\text { slovaque }\end{array}$ & $-29,5$ & $-52,9$ & 16,3 & $-28,6$ & 78,9 & 21,0 \\
\hline Hongrie & $-27,0$ & $-84,4$ & 35,3 & $-34,1$ & 82,5 & 19,8 \\
\hline
\end{tabular}


ce qu'un résidu négatif signifie que les performances réelles sont inférieures à la performance prédite, alors qu'un résidu positif signifie l'inverse.

Pour faciliter l'interprétation des résultats, les pays sont classés par ordre croissant d'agrégation sociale (corrélation intra-classe [rhô] pour le SSE) (4). Les résidus moyens qui ne diffèrent pas statistiquement de 0 ne sont pas repris.

Les résultats sont éloquents. On constate en effet que, dans tous les pays pour lesquels le résidu moyen diffère statistiquement de 0 :

- les élèves ont une performance inférieure à celle que l'on attend d'eux (les résidus sont négatifs) lorsqu'ils fréquentent des écoles défavorisées et ce, qu'ils soient eux-mêmes favorisés ou défavorisés socialement ;

- les élèves ont une performance supérieure à celle que l'on attend d'eux (les résidus sont positifs) lorsqu'ils fréquentent des écoles favorisées et ce, qu'ils soient eux-mêmes favorisés ou défavorisés socialement ;

- lorsque les élèves fréquentent des écoles dites "socialement mixtes », les résidus sont positifs ou faiblement négatifs selon les pays. Pour les élèves défavorisés, cette situation leur est toujours favorable: la moyenne des résidus est positive dans tous les pays. Pour les élèves favorisés, la condition "école socialement mixte " est moins intéressante. La moyenne des résidus est toujours négative.
D'une manière générale, ces données nous conduisent à affirmer la présence d'un effet de la composition socio-économique des écoles sur les performances en science des élèves dans la plupart des pays. L'importance de ces effets d'agrégation varie selon les pays. Ainsi, ils sont quasi inexistants dans des pays comme la Finlande, I'Islande, la Norvège, la Suède et la Pologne. Ils sont importants dans la plupart des autres pays et, en particulier, en Hongrie, Pays-Bas, Allemagne, Autriche, France, République tchèque, Belgique, Italie, Japon, Grèce, Slovaquie, Suisse, Corée, Portugal, Luxembourg, États-Unis, Royaume-Uni, Canada et Australie. Les résultats obtenus ici confirment largement nos analyses précédentes (Crahay \& Monseur, 2006).

\section{Effets des politiques d'agrégation sur l'équité et l'efficacité des systèmes éducatifs}

Maintenant que les conséquences des phénomènes d'agrégation sociale et/ou académique sur les performances des élèves sont documentées, il importe d'en étudier les effets sur l'équité des systèmes éducatifs. L'hypothèse 2 a postule que, plus un système recourt au regroupement social ou académique homogène des élèves, plus la dispersion des performances des élèves sera élevée. Le tableau 2 présente, au niveau pays, les corrélations entre coefficients d'agrégation sociale et écarts-types pour les trois domaines évalués par PISA, tant en 2000 qu'en 2003 et en 2006. Le tableau 3 présente, quant à lui, les résultats des

Tableau 2. - Corrélations entre agrégation sociale et écart type de la performance des élèves

\begin{tabular}{|l|c|c|c|}
\hline & $\begin{array}{c}\text { Agrégation sociale } \\
\text { (PISA 2000) }\end{array}$ & $\begin{array}{c}\text { Agrégation sociale } \\
\text { (PISA 2003) }\end{array}$ & $\begin{array}{c}\text { Agrégation sociale } \\
\text { (PISA 2006) }\end{array}$ \\
\hline Écart type en lecture & 0,02 & 0,22 & 0,42 \\
\hline Écart type en mathématique & 0,49 & 0,16 & 0,51 \\
\hline Écart type en science & 0,29 & $-0,02$ & $-0,16$ \\
\hline
\end{tabular}

Tableau 3. - Corrélations entre agrégation académique et écart type de la performance des élèves

\begin{tabular}{|l|c|c|c|}
\hline & $\begin{array}{c}\text { Agrégation académique } \\
\text { en lecture } \\
\text { PISA 2000 }\end{array}$ & $\begin{array}{c}\text { Agrégation académique } \\
\text { en mathématique } \\
\text { PISA 2003 }\end{array}$ & $\begin{array}{c}\text { Agrégation académique } \\
\text { en science } \\
\text { PISA 2006 }\end{array}$ \\
\hline Écart type en lecture & 0,04 & 0,26 & 0,53 \\
\hline Écart type en mathématique & 0,55 & 0,54 & 0,67 \\
\hline Écart type en science & 0,37 & 0,33 & 0,09 \\
\hline
\end{tabular}


mêmes analyses, mais en fonction de l'agrégation académique.

De ces résultats, se dégage une tendance en demiteinte. La plupart des coefficients de corrélation sont positifs et élevés, témoignant ainsi que, plus un pays regroupe les élèves selon des critères sociaux et/ou académiques, plus la dispersion des résultats est importante, en d'autres termes, plus l'écart qui sépare les élèves faibles des élèves forts s'accroît. Cependant, plusieurs coefficients de régression ne diffèrent pas sensiblement de 0 . Le lien entre phénomènes d'agrégation et disparité des performances scolaires n'est pas aussi systématique que le supposait notre hypothèse $2 a$ qui, sans être réfutée, n'est pas pleinement confirmée.

Nous avons également formulé l'hypothèse que les pratiques de regroupement social ou académique homogène devraient accroître la différence de performance entre les élèves de SSE supérieurs et les élèves à SSE inférieurs (hypothèse $2 b$ ). Pour confirmer cette hypothèse, nous avons calculé la différence de rendement en compréhension de lecture, en culture mathématique et en culture scientifique entre les $25 \%$ d'élèves les plus défavorisés et les $25 \%$ les plus favorisés. Le tableau 4 présente la corrélation entre l'importance des phénomènes d'agrégation sociale et académique et la différence de rendement entre ces deux groupes d'élèves.
Ces résultats confirment l'hypothèse $2 b$ : toutes les corrélations sont positives, ce qui signifie que, plus un système éducatif regroupe les élèves selon des critères académiques et/ou sociaux, plus la différence de rendement entre élèves défavorisés et élèves favorisés est importante. Cet accroissement de la différence entre élèves favorisés et élèves défavorisés semble davantage dépendre de l'agrégation sociale que de l'agrégation académique, en tout cas en compréhension en lecture et en culture scientifique. Or, pour rappel, l'hypothèse $2 \mathrm{c}$ suppose un renforcement du déterminisme social par les phénomènes d'agrégation sociale et académique. Vu les résultats précédents, il est probable que cette dernière hypothèse soit confirmée.

Depuis Bloom (1976) au moins, la corrélation entre SSE et performance scolaire est un indicateur classique du déterminisme social du devenir scolaire des individus. Dans le tableau 5, sont présentées les corrélations entre cet indicateur (lien entre SSE et performance) et les coefficients d'agrégation sociale et académique. Toutes sont positives, ce qui atteste de ce que le déterminisme social est amplifié par les phénomènes d'agrégation, comme nous en faisions I'hypothèse $(2 \mathrm{c})$. Cependant, contrairement à notre hypothèse 2c, l'effet de l'agrégation académique semble moins important que celui de l'agrégation sociale, si l'on en juge par les coefficients de corrélation moins élevés dans un cas que dans l'autre.

Tableau 4. - Corrélations entre agrégation académique et sociale et différences de rendement entre élèves défavorisés et élèves favorisés

\begin{tabular}{|l|c|c|c|}
\hline \multirow{2}{*}{} & \multicolumn{2}{|c|}{ Différences de rendement entre élèves défavorisés et élèves favorisés en } \\
\cline { 2 - 4 } & $\begin{array}{c}\text { Compréhension } \\
\text { en lecture } \\
\text { (PISA 2000) }\end{array}$ & $\begin{array}{c}\text { Culture mathématique } \\
\text { (PISA 2003) }\end{array}$ & $\begin{array}{c}\text { Culture scientifique } \\
\text { (PISA 2006) }\end{array}$ \\
\hline Agrégation académique & 0,33 & 0,66 & 0,44 \\
\hline Agrégation sociale & 0,44 & 0,47 & 0,52 \\
\hline
\end{tabular}

Tableau 5. - Corrélations entre coefficients d'agrégation académique et sociale et coefficient de corrélation entre origine socioculturelle et performance scolaire pour les trois études PISA

\begin{tabular}{|l|c|c|c|}
\hline & $\begin{array}{c}\text { Compréhension } \\
\text { en lecture } \\
\text { PISA 2000 }\end{array}$ & $\begin{array}{c}\text { Culture mathématique } \\
\text { PISA 2003 }\end{array}$ & $\begin{array}{c}\text { Culture scientifique } \\
\text { PISA 2006 }\end{array}$ \\
\hline Agrégation académique & 0,36 & 0,44 & 0,40 \\
\hline Agrégation sociale & 0,60 & 0,57 & 0,70 \\
\hline
\end{tabular}


Tableau 6. - Corrélations entre agrégation sociale et académique et moyenne de la performance des élèves du pays

\begin{tabular}{|l|l|c|c|c|}
\hline & & $\begin{array}{c}\text { Rendement } \\
\text { en lecture }\end{array}$ & $\begin{array}{c}\text { Rendement } \\
\text { en mathématique }\end{array}$ & $\begin{array}{c}\text { Rendement } \\
\text { en science }\end{array}$ \\
\hline \multirow{4}{*}{ Agrégation sociale } & (PISA 2000) & $-0,60$ & $-0,57$ & $-0,50$ \\
\cline { 2 - 5 } & (PISA 2003) & $-0,56$ & $-0,43$ & $-0,31$ \\
\cline { 2 - 5 } & (PISA 2006) & $-0,61$ & $-0,57$ & $-0,51$ \\
\hline \multirow{3}{*}{ Agrégation académique } & en lecture (PISA 2000) & $-0,43$ & $-0,22$ & $-0,32$ \\
\cline { 2 - 5 } & en mathématique (PISA 2003) & $-0,35$ & -003 & $-0,10$ \\
\cline { 2 - 5 } & en science (PISA 2006) & $-0,32$ & $-0,10$ & $-0,10$ \\
\hline
\end{tabular}

Par quels mécanismes les systèmes qui pratiquent le regroupement des élèves en fonction de leur origine sociale produisent-ils cette amplification du déterminisme social ? C'est ce qu'il conviendra d'investiguer plus à fond à l'avenir. Sans doute, cet effet d'amplification passe-t-il par des pratiques pédagogiques ségrégatives dans des pays tels que la Belgique ou la France, mais il semble bien qu'ailleurs, d'autres mécanismes soient à l'œuvre.

Jusqu'ici, nos analyses ont permis de bien documenter la relation entre les phénomènes d'agrégation et l'équité des systèmes éducatifs. Mais nous avons fait l'hypothèse que les mécanismes structurels de production des inégalités sociales avaient aussi des conséquences négatives en termes de rendement (hypothèse 3). Les données rassemblées dans le tableau 6 permettent de confirmer cette hypothèse. On y trouve les corrélations (calculées au niveau pays) entre agrégation sociale, d'abord, agrégation académique, ensuite et la moyenne nationale des performances des élèves.

Comme nous en faisons l'hypothèse, les systèmes éducatifs qui pratiquent l'agrégation académique ou sociale ont tendance à être moins efficaces et ce, dans tous les domaines. En effet, toutes les corrélations sont négatives. Notons également que celles qui lient le rendement à l'agrégation sociale sont plus fortes que celles qui concernent l'agrégation académique. Dans l'ensemble, les résultats indiquent que, plus les pays disposent d'établissements scolaires concentrant des populations homogènes du point de vue du statut socio-économique, plus le rendement moyen du pays est faible. En d'autres termes, les pays qui ont adopté une politique éducative qui minimise les regroupements homogènes des élèves selon des critères académiques ou sociaux se caractérisent, en moyenne, par des rendements moyens supérieurs que les pays qui ont opté pour ou laissé s'installer la ségrégation. II est donc possible d'être efficace et en même temps, équitable.

\section{Conclusions et discussion}

Les analyses secondaires de PISA 2006 présentées ici illustrent le potentiel de l'éducation comparée. Le regard qui est posé par le chercheur à travers les différents systèmes d'enseignement, s'il s'appuie sur des modèles statistiques appropriés, appliqués à des données de qualité, permet de faire émerger les ressemblances entre systèmes éducatifs et, complémentairement, de débusquer leurs spécificités. II permet ainsi de montrer que certaines caractéristiques structurelles des systèmes d'enseignement influent sur les performances des élèves.

Le poids de l'origine sociale des élèves sur leurs performances académiques doit être affirmé une fois de plus. Cependant, l'influence des caractéristiques des écoles et, plus particulièrement, de leur composition sociale et académique, est également très importante ; il semble même légitime de se demander dans quelle mesure cette dimension n'est pas plus importante que le SSE en tant que variable individuelle. II y a bien un effet école et celui-ci est tributaire des caractéristiques des systèmes éducatifs. Tous les résultats engrangés ici, mais aussi par Crahay \& Monseur (2006), vont dans le même sens. Cet effet école est aussi et, avant tout, pensons-nous, un effet d'agrégation sociale et académique.

Cette conjugaison des phénomènes d'agrégation sociale et d'agrégation académique semble fluctuer 
selon les pays. C'est ce qui tend à se dégager des analyses présentées ci-dessus. Plus largement, les analyses successives présentées ici indiquent, d'une part, combien les processus générateurs d'inégalités sont intriqués et, d'autre part, que ceux-ci sont partiellement ordonnés. En effet, comme nous l'avons rappelé ci-dessus, il semble bien que l'influence de la variable immigration comme celle de la variable " langue parlée à la maison » soit " engloutie » dans l'effet exercé par le statut socio-économique et culturel de la famille d'origine des élèves. Ce constat a son importance en matière de politique de lutte contre les inégalités de réussite à l'école : en cherchant et en trouvant les moyens de juguler les effets préjudiciables de l'origine sociale, on agit du même coup sur les inégalités liées à l'immigration et à la langue parlée à la maison.

Or, eu égard à la convergence des résultats de recherches, y compris nos diverses analyses, pour mettre en évidence l'importance des phénomènes d'agrégation sur la réussite scolaire, il y a là, assurément, plus qu'une piste à explorer. II y a là un fait qui nous paraît désormais bien établi. Notre contribution consiste notamment à confirmer la généralité et quasiment l'universalité des effets pervers des phénomènes d'agrégation. L'ampleur de ces phénomènes, ainsi que celle de leurs incidences négatives, varie selon les pays, mais leur caractère préjudiciable est partout avéré. Dès lors que des élèves se retrouvent dans des écoles recrutant majoritairement des enfants d'origine sociale " défavorisée ", leurs performances académiques sont inférieures à ce qu'elles pourraient être s'ils fréquentaient une école brassant les origines sociales. Bref, les phénomènes d'agrégation ont des effets négatifs sur les individus. Ils ont également des conséquences négatives au niveau système.

Le regroupement par établissement d'élèves aux caractéristiques sociales et académiques similaires se traduit par des ségrégations entre élèves de catégories sociales différentes qui ne s'avèrent bénéfiques ni pour les individus qui se retrouvent dans les écoles défavorisées, ni pour les systèmes. Les analyses présentées ici montrent clairement que, plus les systèmes d'enseignement sont marqués par les phénomènes d'agrégation, plus la dispersion des performances est élevée et plus leur moyenne au niveau du pays est faible.

Cette convergence de résultats ainsi que leur généralité et leur réplication doivent donner à réfléchir aux responsables des systèmes éducatifs. II nous semble que le doute n'est plus permis : pour réduire l'ampleur des inégalités de réussite à l'école et pour accroître la rentabilité générale des systèmes éducatifs, il convient d'endiguer autant que faire se peut les phénomènes d'agrégation.

Par quelles voies mener cette politique éducative ? Pour répondre avec précision à cette question, il est nécessaire de saisir au mieux comment ces processus se génèrent. Sans entrer dans le détail des situations nationales, il est manifeste que ceux-ci mettent notamment en œuvre l'affectation des élèves aux écoles et la diversité des types d'écoles composant l'offre éducative. Autrement dit, l'équité et l'efficacité en matière de performances scolaires sont indissolublement liées à la gestion de I'hétérogénéité des élèves. En favorisant celle-ci au niveau des écoles et des classes, il est possible de lutter efficacement contre les inégalités de réussite scolaire et, probablement par voie de conséquence, professionnelle. Les bons résultats obtenus aux enquêtes PISA par les pays dans lesquels la majorité des écoles peut se targuer de la mixité sociale de leur public en sont la preuve.

Christian Monseur cmonseur@ulg.ac.be

Professeur à l'université de Liège Directeur du service d'approches quantitatives des faits éducatifs

Marcel Crahay Marcel.Crahay@pse.unige.ch ou Marcel.Crahay@ulg.ac.be Professeur aux universités de Genève et de Liège en sciences de l'éducation

\section{NOTES}

(1) Par souci de brièveté, dans la suite du texte, nous utiliserons SSE pour statut socio-économique des élèves.

(2) Dans la perspective de la présente publication, nous avons, en effet, vérifié sur les données PISA 2006 que les constats opérés sur les données PISA 2000 sont confirmés.

(3) Les élèves de chaque école ont été considérés comme un échantillon aléatoire et simple. Un test d'égalité de moyennes a ensuite été réalisé pour déterminer si la moyenne du SSE des élèves d'une école diffère statistiquement ou non de la moyenne nationale.

(4) Mathématiquement, la corrélation intra-classe correspond au pourcentage de variance SSE qui se situe entre établissements scolaires. 


\section{BIBLIOGRAPHIE}

BLOOM B.S. (1976). Human characteristics and school learning. New York: Mc Graw-Hill.

CHERKAOUI M. (1979). Les paradoxes de la réussite scolaire. Paris : PUF.

COLEMAN J.S., CAMPBELL E., HOBSON C., MCPARTLAND J., MOOD A., WEINFELD F. \& YORK R. (1966). Equality of Educational Opportunity. Washington: Government printing office.

CRAHAY M. (2000). L'école peut-elle être juste et efficace? Bruxelles : De Boeck.

CRAHAY M. \& MONSEUR C. (2006). «Différences individuelles et effets d'agrégation en ce qui concerne les performances en lecture. Analyse secondaire des données PISA 2000 ». In C. HOUSSEMAND, R. MARTIN \& P. DICKES, Perspectives de psychologie différentielle. Rennes: Presses universitaires de Rennes, p. 23-34.

CRAHAY M. (2007). Peut-on lutter contre l'échec scolaire? Bruxelles : De Boeck.

DURU-BELLAT M. \& MINGAT A. (1997). « La constitution de classes de niveau par les collèges et ses incidences sur les progressions et les carrières des élèves ". Revue française de sociologie, vol. 38, $\mathrm{n}^{\circ} 4$, p. 759-790.

DURU-BELLAT M. (2003). «Les apprentissages des élèves dans leur contexte: les effets de la composition de l'environnement scolaire ». Carrefours de l'éducation, vol. 16, p. 182-206.
GRISAY A. (1993). "Le fonctionnement des collèges et ses effets sur les élèves de sixième et de cinquième ». Les Dossiers Éducation et formation, $\mathrm{n}^{\circ} 32$. Paris : ministère de l'Éducation nationale, direction de l'Évaluation et de la Prospective.

HUSÉN T. (1975). Influence du milieu social sur la réussite scolaire. Paris : OCDE-CERI

KEEVES J.P. (1992). Changes in science achievement in ten countries. Oxford : Pergamon press.

MAYESKE G.W., WISLER C.-E., BEATON A.-E., WEINFELD F.-D., COHEN W.M., OKADA T., PROSHEK J.M. \& TAE K.A. (1972). A Study of our nation's school. Washington: USOE.

OCDE (2001). Connaissances et compétences : des atouts pour la vie. Premiers résultats de PISA 2000. Paris: OCDE.

OCDE (2004). Apprendre aujourd'hui, réussir demain. Premiers résultats de PISA 2003. Paris: OCDE.

OCDE (2007). PISA 2006. Les compétences en sciences, un atout pour réussir. Paris : OCDE.

VANDENBERGHE V. (2001). " Educational equity: social interactions may matter ». In W. HUTMACHER, D. COCHRANE \& N. BOTTANI, In pursuit of equity in education. Using international indicators to compare equity policies. Dordrecht : Kluwer academic publisher, p. 64-91. 